6. Chou, C.-W. et al. Science 316, 1316-1320 (2007).

Boozer, A. D., Boca, A., Miller, R., Northup, T. E. \& Kimble, H. J. Phys. Rev. Lett. 98, 193601 (2007).
8. Keller, M., Lange, B., Hayasaka, K., Lange, W. \& Walther, H. Nature 431, 1075-1078 (2004).

9. Häffner, H., Roos, C. \& Blatt, R. Phys. Rep. 469, 155-203 (2008).
10. Barros, H. G. et al. New J. Phys. 11, 103004 (2009).

11. Steiner, M., Meyer, H. M., Deutsch, C., Reichel, J. \& Köhl, M. Phys. Rev. Lett. 110, 043003 (2013).

\title{
RESEARCH
}

\section{New centre, new vision}

14 December 2012 saw the official launch of the Centre for Disruptive Photonic Technologies (CDPT) at Nanyang Technological University (NTU), Singapore. The CDTP makes up a major component of NTU's Photonics Institute, which is a joint research institute between the Optoelectronics Research Centre (ORC) of the University of Southampton and NTU.

Netting seed funding of $\$ \$ 10$ million from the Ministry of Education (MOE), Singapore, and $\$ \$ 6.2$ million from the university, the centre, established in October 2012, is expecting to attract substantial additional funding from industry, as well as local and international agencies. It has also secured $\$ \$ 2.1$ million from the Agency of Science, Technology and Research ( $A^{\star} S T A R$ ) in Singapore.

The research interests of the centre range from few-photon switching devices, cognitive nanophotonics and novel nanophotonic materials, through to reconfigurable, dynamic and quantum metamaterials, plasmonic and nanofibre waveguides, nanolasers and spasers.

"In short, our core agenda is disruptive ideas in nanophotonics," summarized Nikolay Zheludev, director of the CDTP and deputy director of the ORC. "By advancing the physics of the control, guidance and amplification of light in nanostructures and by developing new nanofabrication techniques and methods of growth, hybridization and integration into the waveguide and fibre environment of different novel material structures, we aim to develop and test groundbreaking, disruptive concepts that could find applications in photonic devices," Zheludev elaborated.

The CDPT currently has seven principal investigators and three associate principal investigators, all of whom are NTU staff. It aims to employ ten research fellows by the end of 2013 and to accept 20 research students over the next five years. The CDPT already attracts strong interest from researchers around the world, and it is seen as a hub of collaboration. The centre currently has seven academic

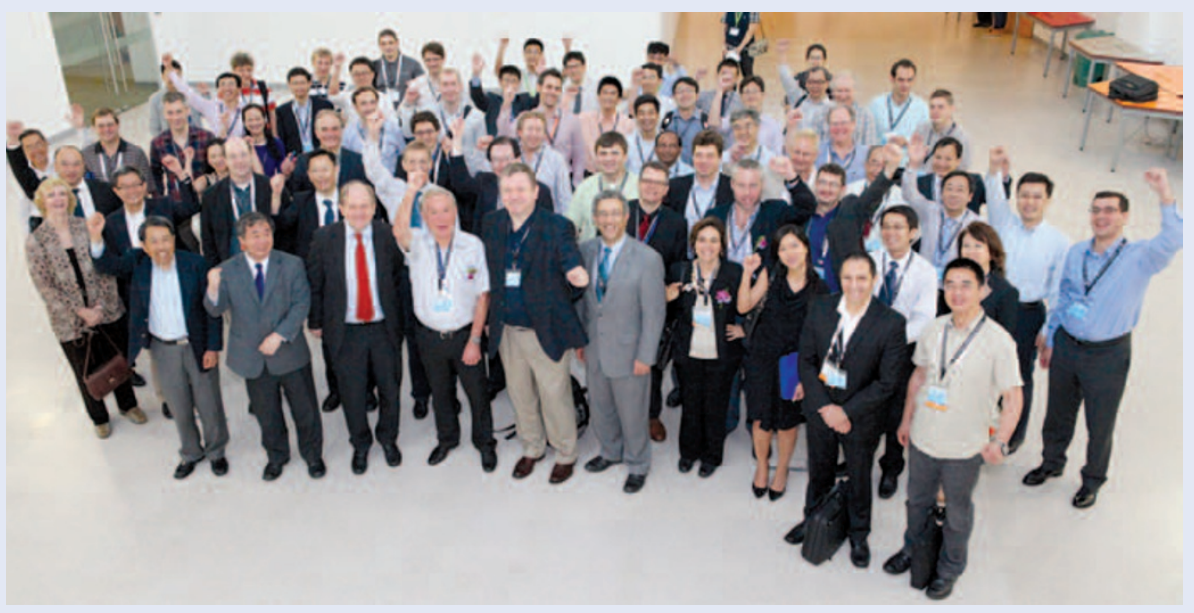

collaborators from the USA, Germany,

Australia, Taiwan and the UK.

"All our collaborators are leaders of large and highly influential research groups. We are setting real joint projects with them and provide mobility support for international exchanges with these groups," said Zheludev. The centre is now working with new partners from France and China, and is exploring a link with Japan. "We are very open to collaboration," he added.

The CDPT has ambitious plans to go much further. "In a grand vision, the CDPT is seen as part of the Photonics Institute at NTU," said Sir David Payne, director of the ORC, when asked about the future outlook of the centre.

"Science and technology are becoming global. To address this challenge, we are creating a world-leading dual research institute for photonics that spans both Singapore and the UK. The Photonics Institute will be a cradle of new knowledge and intellectual property, and a focal point for developing ground-breaking applications of light-enabled technologies," Zheludev followed up.

Swee Chuan Tjin, director of Projects, President's Office at NTU explained that the Photonics Institute will be based in both NTU and the University of Southampton to provide global leadership and consolidation of resources in the research of photonic materials, devices, processes and systems of the future. "By analogy with multinational companies, we will create a world-dominating consortium of equal partners united by common aspirations, shared resources, coherent management and strategic thinking."

When asked about the challenges in making this dual institute a reality, colleagues at NTU and the University of Southampton stressed that academic enthusiasm alone is not sufficient. The Photonics Institute is a radically new concept of equal partnership, which is only possible between institutions that have mutual trust and respect at the highest levels of decision making. There are intellectual property, legal and contractual matters that require attention, but a good working relationship between the two universities at different levels, particularly between NTU president Bertil Andersson and University of Southampton vicechancellor Don Nutbeam, forms the basis for success.

"We are working on these matters in a spirit of collaboration," Payne, Zheludev and Tjin concluded.

As can be imagined, forming a centre that involves different institutions can be very challenging, especially when they are in two different countries. However, good things come to those who act.

RACHEL WON 